\title{
Potential oscillations near a barrier in the presence of phase-breaking scattering
}

\author{
E. Tekman and S. Ciraci \\ Department of Physics, Bilkent University, Bilkent 06533, Ankara, Turkey \\ (Received 31 July 1991; revised manuscript received 30 September 1991)
}

\begin{abstract}
Using the Green's function method for nonequilibrium processes, we study the potential oscillations near a barrier in both coherent- and incoherent-transport regimes. In the fully coherent regime the local electrochemical potential oscillates near the barrier, due to interference of the incident and reflected waves. The inclusion of phase-breaking scattering leads to suppression of these oscillations as a result of increasing contribution from the incoherent processes. As one goes away from the barrier, the amplitude of oscillations is found to decay with a decay length equal to the phase-scattering length.
\end{abstract}

Structures across which the electron wave function retains its phase are commonly denoted as mesoscopic systems. ${ }^{1}$ In recent years, experiments on electron waveguides and quantum-point contacts unambiguously showed that at such small length scales electron transport can thoroughly be understood only by use of the quantum-mechanical wave function and especially including the effects of its phase. In basic quantum mechanics a particle is assumed to perform wave motion throughout space without suffering from scattering events which randomly change its phase (i.e., phasebreaking scattering). Since the early days of studying mesoscopic systems it has been stressed that one has to go beyond this naive approach for a complete interpretation of the experimental data. To this end, a phasecoherence length $L_{\phi}$, which is the average distance traveled by electrons without having a phase-breaking scattering, has been introduced. ${ }^{2}$ Accordingly, the interference effects are present only when the size of the device is on the order of $L_{\phi}$, but diminish as the size gets larger.

The effects of phase-breaking scattering have been included in the ensemble-averaged properties using diagrammatic techniques ${ }^{3}$ based on the Kubo formalism. The study of dynamics and kinetics of the system in the presence of phase breaking, on the other hand, is only recent. Datta ${ }^{4}$ employed the Dyson equation in the Keldysh formalism ${ }^{5}$ to derive a steady-state quantum kinetic equation in the presence of inelastic scattering. His formulation is applicable to the phase-breaking scattering as well, for which energy transfer between the electron and its environment is not necessary. Results of Datta showed that an excitation travels a distance on the order of $L_{\phi}$ before losing its phase information, indicating that the Keldysh formalism is appropriate for studying transport in the mesoscopic regime. D'Amato and Pastawski ${ }^{6}$ studied the effects of dephasing using the multiprobe generalization of the Landauer formula as proposed by Büttiker. ${ }^{7}$ According to Büttiker's approach the voltage probes in the circuit are sources of inelastic scattering since they are connected to macroscopic reservoirs and thus the incoming and outgoing fluxes are totally incoherent. Reversing this statement one finds that inelastic scattering in a device can be modeled by using ficti- tious voltage probes. $^{8}$ In fact, the equivalence of the multiprobe approach to dephasing has recently been shown. ${ }^{9}$ In their work, D'Amato and Pastawski focused attention on the conductance of a disordered linear chain for which elastic scattering and localization are as important as inelastic scattering. Recently Flores and Anda ${ }^{10}$ also used the Keldysh formalism ${ }^{5}$ to study the conductance of a linear chain by including inelastic-scattering events.

The subject matter of this paper is a more direct study of phase coherence in mesoscopic systems. It is known ${ }^{11}$ that the local chemical potential oscillates near a barrier in the presence of transport due to interference of incident and reflected waves. Büttiker ${ }^{11}$ examined the relevance of Landauer formulas by studying these potential oscillations. Oscillations of the same nature were shown ${ }^{12}$ to exist in narrow constrictions as well. It was argued that the potential oscillations may also affect the noninvasive measurements by the scanning-tunneling microscope. ${ }^{13}$ In this paper we study the effect of phasebreaking scattering on the potential oscillations. First, we apply the Keldysh formulation ${ }^{5}$ for nonequilibrium processes to calculate carrier density and current in a mesoscopic system. We use a strictly one-dimensional (1D) model and a local self-energy operator, ${ }^{4}$ and find that the chemical potential oscillations are suppressed as a result of phase-breaking scattering. Moreover, we show that the strength of such interference effects decay as $\exp \left(-l / L_{\phi}\right)$, where $l$ is the total distance to be traveled by the electron wave in order to give rise to interference (i.e., twice the distance from the point of measurement to the barrier for the present problem).

We first follow the approach developed by Datta, ${ }^{4}$ and we start with the Dyson equation in the Keldysh formulation, ${ }^{4,5}$ in terms of the Hamiltonian of the noninteracting system $H_{0}$, Green's function $\widetilde{G}$, and self-energy $\widetilde{\Sigma}$ matrices. Assuming a steady-state condition, the quantumkinetic equation for the Green's function

$$
\begin{aligned}
& {\left[E-H_{0}(\mathbf{r})\right] \widetilde{G}\left(\mathbf{r}, \mathbf{r}^{\prime} ; E\right)} \\
& =\delta\left(\mathbf{r}-\mathbf{r}^{\prime}\right) \widetilde{I}+\int d \mathbf{r}^{\prime \prime} \widetilde{\Sigma}\left(\mathbf{r}, \mathbf{r}^{\prime \prime} ; E\right) \widetilde{G}\left(\mathbf{r}^{\prime \prime}, \mathbf{r}^{\prime} ; E\right)
\end{aligned}
$$

is obtained from the Fourier transform of the Dyson equation. Furthermore, this differential equation can be transformed into an integral equation 


$$
\begin{aligned}
\widetilde{G}\left(\mathbf{r}, \mathbf{r}^{\prime} ; E\right)=\widetilde{G}_{0}\left(\mathbf{r}, \mathbf{r}^{\prime} ; E\right)+\int & d \mathbf{r}^{\prime \prime} d \mathbf{r}^{\prime \prime \prime} \widetilde{G}_{0}\left(\mathbf{r}, \mathbf{r}^{\prime \prime} ; E\right) \\
& \times \widetilde{\Sigma}\left(\mathbf{r}^{\prime \prime}, \mathbf{r}^{\prime \prime \prime} ; E\right) \widetilde{G}\left(\mathbf{r}^{\prime \prime \prime} ; E\right)
\end{aligned}
$$

by using the Green's function for the noninteracting system $\widetilde{G}_{0}\left(\mathbf{r}, \mathbf{r}^{\prime} ; E\right)$, which satisfies the equation $\left(E-H_{0}\right) \widetilde{G}_{0}=\widetilde{I}$. The electron density $n(\mathrm{r} ; E)$, hole density $p(\mathbf{r} ; E)$, and current density $\mathbf{j}(\mathbf{r} ; E)$ (all per unit energy), in turn, can be found in terms of the Green's function. Details of the derivation are the same as those found in Ref. 4. For an arbitrary self-energy function, Eq. (2) is difficult to solve and does not have any advantages over the quantum-kinetic equation Eq. (1). However, with the following assumptions it can be simplified to a great extent. First, we restrict our attention to a strictly $1 \mathrm{D}$ system. Second, we include the elastic-scattering events into $\widetilde{\Sigma}$ together with phase-breaking scattering. This way $\widetilde{G}_{0}\left(x, x^{\prime} ; E\right)$ can be calculated directly from a 1D free-electron approximation. While doing so we assume that the device is connected to two reservoirs at $x= \pm \infty$ having chemical potentials $\mu_{L}$ and $\mu_{R}$ for the left- and right-hand-side reservoir, respectively. Clearly, we do not impose any boundary conditions on the Green's function on a boundary specified $a$ priori. This is consistent with the open nature of the system at hand. ${ }^{14}$ That is, the system is driven by external agents and the response of the system to this external excitation can be found only after completely solving the problem. Lastly we assume that scattering events (both elastic and phase breaking) take place at discrete and uncorrelated scattering center. ${ }^{4}$ To this end, one can write

$$
\widetilde{\Sigma}\left(x, x^{\prime} ; E\right)=\delta\left(x-x^{\prime}\right) \sum_{i} \widetilde{\Sigma}_{i}(E) \delta\left(x-x_{i}\right),
$$

where $\widetilde{\Sigma}_{i}(E)$ denotes the self-energy contribution by the $i$ th scatterer located at $x_{i}$.

Substituting Eq. (3) into Eq. (2) one can solve $\widetilde{G}$ in terms of $\widetilde{G}_{0}$. Then, the electron density and current density are calculated in terms of the noninteracting Green's function and the reducible self-energy matrix

$$
\widetilde{\Sigma}_{r}\left(x, x^{\prime} ; E\right)=\sum_{i j}\left[\widetilde{\Sigma}_{r}(E)\right]_{i j} \delta\left(x-x_{i}\right) \delta\left(x^{\prime}-x_{j}\right)
$$

as

$$
\begin{aligned}
n(x ; E)= & n_{0}(x ; E)+\frac{1}{\pi} \operatorname{Im}\left(\sum_{i, j} G_{0}^{R}\left(x, x_{i} ; E\right)\left[\Sigma_{r}^{R}(E)\right]_{i j} G_{0}^{<}\left(x_{j}, x ; E\right)\right]-\frac{i}{2 \pi} \sum_{i j} G_{0}^{R}\left(x, x_{i} ; E\right)\left[\Sigma_{r}^{<}(E)\right]_{i j} G_{0}^{A}\left(x_{j}, x ; E\right), \\
j(x ; E)= & j_{0}(x ; E)-\frac{\hbar}{2 \pi m} \operatorname{Re}\left[\sum_{i, j} \frac{\partial G_{0}^{R}\left(x, x_{i} ; E\right)}{\partial x}\left[\Sigma_{r}^{R}(E)\right]_{i j} G_{0}^{<}\left(x_{j}, x ; E\right)\right] \\
& -\frac{\hbar}{2 \pi m} \operatorname{Re}\left(\sum_{i, j} G_{0}^{R}\left(x, x_{i} ; E\right)\left\{\left[\Sigma_{r}^{<}(E)\right]_{i j} \partial G_{0}^{A}\left(x_{j}, x ; E\right) / \partial x+\left[\Sigma_{r}^{R}(E)\right]_{i j} \partial G_{0}^{<}\left(x_{j}, x ; E\right) / \partial x\right\}\right) .
\end{aligned}
$$

Here superscripts $<,>, A$, and $R$ stand for the electron, hole, advanced, and retarded functions, respectively. Note that, the reducible self-energy matrix satisfies the Dyson equation $\widetilde{\Sigma}_{r}=\widetilde{\Sigma}+\widetilde{\Sigma} \widetilde{G}_{0} \widetilde{\Sigma}_{r}$.

In order to study potential oscillations in the presence of phase-breaking scattering, we assume that $\widetilde{\Sigma}_{i}$ of all the scatterers has a phase-breaking part, but only selfenergies of the scatterers lying in the barrier region have an elastic-scattering part. The phase-breaking part is given by

$$
\begin{aligned}
& \Sigma_{i}^{<}(E)=\left[i \hbar / \tau_{i}(E)\right] p\left(x_{i} ; E\right), \\
& \Sigma_{i}^{>}(E)=\left[-i \hbar / \tau_{i}(E)\right] n\left(x_{i} ; E\right) .
\end{aligned}
$$

As shown in Ref. 4 these self-energy functions are consistent with the golden-rule expression for a system of electrons interacting with a reservoir of oscillators at thermodynamic equilibrium, with the restriction that the scattering is not inelastic (i.e., the energy of the electron is conserved). In addition, one can show that the selfenergies given by Eq. (5) yield a conserved current throughout the device. This point can also be reached starting from the multiprobe approach and yields Ward identities. ${ }^{9}$ Note that Eq. (5) has a simple physical interpretation as well. Taking $\tau_{i}(E)$ as the average time for an electron or a hole of energy $E$ to suffer a phasebreaking scattering at site $i$, assuming that the state (elec- tron or hole state) is initially filled, $\Sigma_{i}^{<}(E)$ and $\Sigma_{i}^{>}(E)$ become the average times between these scattering events including the effects of exclusion. One other important point to notice is that the self-energy functions depend on the electron and hole densities. Therefore, Eqs. (4) and (5) have to be solved self-consistently. The elastic part of the self-energies, on the other hand, is given by $\sigma_{i}(E) \widetilde{I}$, and is independent of the carrier densities.

Finally, we define a local electrochemical potential within the linear-response approximation, i.e., by neglecting all the energy dependences and calculating everything at the Fermi level $E_{F}$. To this end we consider a reservoir at thermodynamic equilibrium with chemical potential $\mu_{i}$, connected to the $i$ th scattering site by an ideal wire ${ }^{15}$ (i.e., without any internal structure ). Assuming that $\mu_{L}>\mu_{R}$ one finds that the current into the reservoir is proportional to $\left(\mu_{L}-\mu_{i}\right) n\left(x_{i} ; E_{F}\right)$, and the current out of the reservoir is proportional to $\left(\mu_{i}-\mu_{R}\right) p\left(x_{i} ; E_{F}\right)$, the proportionality constants being the same. Thus, in order to have zero net current into the reservoir its chemical potential has to be given by

$$
\mu_{i}=\frac{\mu_{L} n\left(x_{i} ; E_{F}\right)+\mu_{R} p\left(x_{i} ; E_{F}\right)}{n\left(x_{i} ; E_{F}\right)+p\left(x_{i} ; E_{F}\right)} .
$$

This definition of $\mu_{i}$ is reminiscent of the counting argument of Landauer. ${ }^{14}$ However, in the present study the 


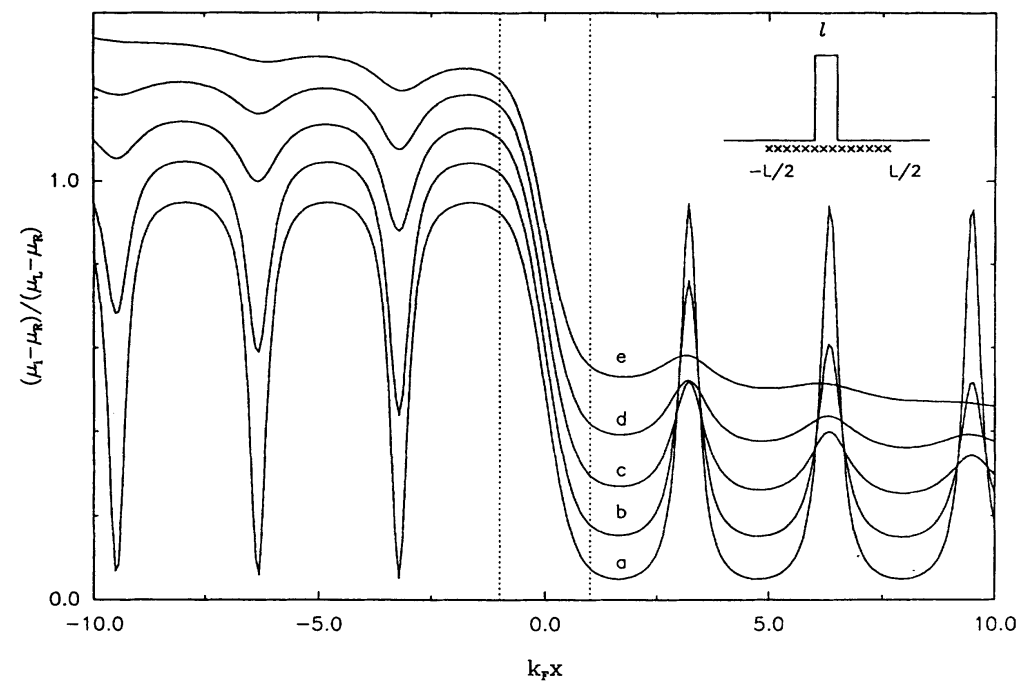

FIG. 1. Variation of the local electrochemical potential profile for $L / L_{\phi}$ values of $a, 0 ; b$, $0.1 ; c, 0.5 ; d, 1 ; e, 2$. Dotted lines indicate the position of the potential barrier with $k_{F} l=2$, and $\sigma=0.06 \times \hbar^{2} k_{F} / m$. The length of the device is $k_{F} L=20$, and the density of scatterers is $\rho_{\mathrm{sc}}=12.5 k_{F}$. The curves are offset by 0.1 for clarity. Inset schematically shows the structure, the cross-shaded portion denoting the region in which phase-breaking scattering takes place.

carrier densities are determined from full quantummechanical calculations and have interference properties. ${ }^{11,16}$ As shown by Büttiker, ${ }^{16} \mathrm{Eq}$. (6) has to be corrected to include the self-consistent potential if the Fermi wavelength is shorter than or comparable to the screening length. Note that $\mu_{i}$ is not a real electrochemi-
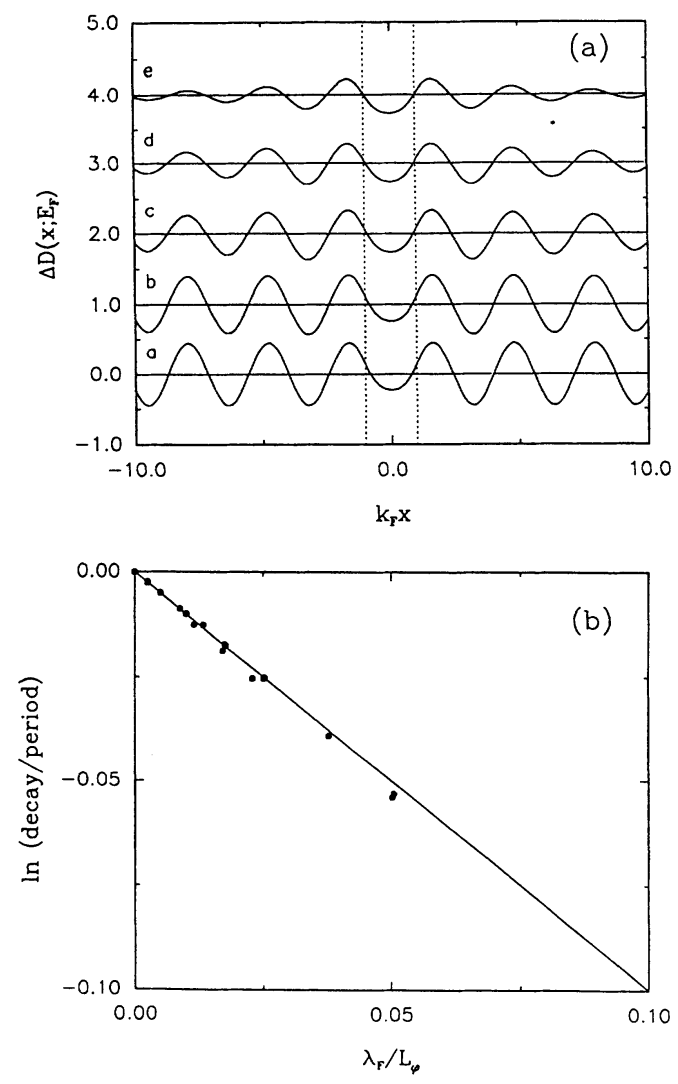

FIG. 2. (a) Percent change in the total density of states $\Delta D\left(x ; E_{F}\right)$, corresponding to the potential profiles in Fig. 1 . The curves are offset by 1 . (b) Decay of the oscillation amplitude of $\Delta D\left(x ; E_{F}\right)$ per period as a function of $\lambda_{F} / L_{\phi}$. The filled circles are averages over elastic-scattering strength values $\sigma$, for various values of $\rho_{\mathrm{sc}}, l$, and $L$. The solid line denotes $\exp \left(-\lambda_{F} / L_{\phi}\right)$. cal potential since it does not determine the carrier density at that under local thermodynamic equilibrium conditions. ${ }^{4}$

At this point, it is in order to compare the present work with those proposed earlier. Our work uses Datta's approach, ${ }^{4}$ but differs from it in the following points: Instead of solving the differential kinetic equation (as Datta did) we solve the integral equation $\mathrm{Eq}$. (2) for the interacting Green's function. This provides significant advantages. (i) For a local self-energy function the Dyson equation reduces to a set of matrix equations. Consequently the interacting Green's function can be calculated by using the noninteracting Green's function and reducible self-energy function. The self-consistency scheme becomes transparent and numerically tractable in this way. (ii) We include the external current through the circuit in the noninteracting Green's function $\widetilde{G}_{0}$, so that it is not necessary to introduce heuristic external sources. In addition, the artifacts due to the $a$ priori selected position of the contacts are eliminated. In spite of the fact that a simple model for the current sources may be employed $^{17}$ within the current model, we believe that the effect of the probes is more subtle and has to be investigated separately.

The local electrochemical potential used in the present work and Datta's method ${ }^{4}$ have different definitions. Datta assumes local thermodynamic equilibrium and defines the local potential accordingly. In the present work, however, the local electrochemical potential is obtained as a result of a noninvasive measurement. That is, there is no need to have thermodynamic equilibrium, even locally. The present definition may be used for cases beyond linear response as well, since it depends on the total interaction between the system and the noninvasive voltage probe. The linear-response assumption is introduced to simplify the solution, and is not essential.

The method of Flores and Anda ${ }^{10}$ uses the tightbinding model and starts with two disconnected bodies at thermodynamic equilibrium of different chemical potentials and obtains the nonequilibrium Green's function $\widetilde{G}_{0}$ by connecting these two systems. In our method, as described above, we assume that there is an a priori non- 
equilibrium distribution of carriers for the noninteracting system and we calculate $\widetilde{G}_{0}$ accordingly. The multiprobe approaches, ${ }^{6,9}$ on the other hand, are aiming only at the calculation of the conductance with the help of artificial dephasing probes. Thus, they may not be appropriate for more detailed analyses. More recently, McLennan and co-workers ${ }^{18}$ also used the formalism developed by Datta to study voltage drop in mesoscopic systems. Their work and ours ${ }^{17,19}$ independently arrived at the same conclusion that the phase-breaking scattering leads to decaying potential oscillations going away from an obstacle.

We applied the present method to the structure shown in the inset to Fig. 1. This structure is characterized by three parameters: the phase-breaking time $\tau$, elasticscattering strength $\sigma$ (both are calculated at $E_{F}$ and are the same for all scatterers), and the density of scatterers $\rho_{\text {sc }}$. The barrier extends from $-l / 2$ to $+l / 2$, and the phase-breaking scatterers are uniformly distributed between $-L / 2$ and $+L / 2$. The phase-breaking length $L_{\phi}$ is given by

$$
L_{\phi}=\hbar k_{F} \tau / m \rho_{\mathrm{sc}},
$$

which is the Fermi velocity times the phase-breaking time averaged over the unit length. In Fig. 1 the local electrochemical potential profile is shown for the varying $L / L_{\phi}$ ratio. Clearly, for $L_{\phi} \rightarrow \infty$ one obtains the phasecoherent result ${ }^{11}$ and potential oscillations have the same oscillation amplitude independent from the distance to the barrier. In the other extreme, i.e., for $L_{\phi}<<$, phase coherence is lost and electrons act as classical particles. Thus, out of the barrier region $\mu_{i}$ varies linearly with position. ${ }^{6}$ In the intermediate regime potential oscillations are still present, but their amplitudes get smaller as they go away from the barrier. That is, a transition from the classical regime to the quantum regime takes place as a function of $L / L_{\phi}$. One can show by using Eqs. (3) and (2) that the period of oscillations is $\lambda_{F} / 2$ provided that $L_{\phi} \gg \lambda_{F}$.

We develop a more quantitative approach to these decaying oscillations by considering the total density of states per unit energy, ${ }^{4}$

$$
D(x ; E)=n(x ; E)+p(x ; E) .
$$

Note that in the absence of the barrier and phasebreaking scattering, $D\left(x ; E_{F}\right)$ is just equal to the density of states $D_{0}\left(E_{F}\right)=m / \pi \hbar^{2} k_{F}$. In the limit $L_{\phi}<<\lambda_{F}$, on the other hand $D\left(x ; E_{F}\right)$ satisfies a diffusion equation, and hence exponentially decays away from the barrier. The percent change in the density of states

$$
\Delta D\left(x ; E_{F}\right)=\left[D\left(x ; E_{F}\right)-D_{0}\left(E_{F}\right)\right] / D_{0}\left(E_{F}\right),
$$

corresponding to the potential profiles in Fig. 1, is shown in Fig. 2(a). The envelope of $\Delta D\left(x ; E_{F}\right)$ may be compared to the retarded Green's function as found by Dat$\mathrm{ta}^{4}{ }^{4}$ since it represents the propagation of the excitation created by the barrier. In order to analyze the interference effects away from the barrier, in Fig. 2(b) decay of magnitude of $\Delta D\left(x ; E_{F}\right)$ per period is shown as a function of $\lambda_{F} / L_{\phi}$. Clearly, $L_{\phi}$ dependence of decay is given by $\exp \left(-\lambda_{F} / L_{\phi}\right)$. This is due to the fact that in order for incident and reflected waves to interfere a distance $d$ away from the barrier, the wave has to travel a total distance of $2 d$ without suffering a phase-breaking scattering. Consequently, the strength of the interference decays as $\exp \left(-2 d / L_{\phi}\right)$. This result verifies the phenomenological exponential dependence ${ }^{2}$ of the phase-coherence effects on $L_{\phi}$, and corroborates that a diffusion approach is appropriate for studying mesoscopic systems with phasebreaking scattering. ${ }^{3}$
${ }^{1}$ For a review, see Nanostructure Physics and Fabrication, edited by M. A. Reed and W. P. Kirk (Academic, New York, 1989).

${ }^{2}$ B. L. Al'tshuler, A. G. Aronov, and D. E. Khmel'nitskii, Solid State Commun. 39, 619 (1981); J. Phys. C 15, 7367 (1982).

${ }^{3}$ P. A. Lee and A. D. Stone, Phys. Rev. Lett. 55, 1622 (1985); B. L. Al'tshuler and B. I. Shklovskii, Zh. Eksp. Teor. Fiz. 91, 220 (1986) [Sov. Phys. JETP 64, 127 (1986)]; P. A. Lee, A. D. Stone, and H. Fukuyama, Phys. Rev. B 35, 1039 (1986).

${ }^{4}$ S. Datta, Phys. Rev. B 40, 5830 (1989); J. Phys. Condens. Matter 2, 8023 (1990).

${ }^{5}$ L. V. Keldysh, Zh. Eksp. Teor. Fiz. 47, 1515 (1964) [Sov. Phys. JETP 20, 1018 (1965)]. We use the notation of G. D. Mahan, Phys. Rep. 145, 251 (1987).

${ }^{6}$ J. L. D'Amato and H. M. Pastawski, Phys. Rev. B 41, 7411 (1990).

${ }^{7}$ M. Büttiker, Phys. Rev. Lett. 57, 1761 (1986).

${ }^{8}$ H. L. Engquist and P. W. Anderson, Phys. Rev. B 24, 1151 (1981); M. Büttiker, ibid. 33, 3020 (1986).

${ }^{9}$ S. Hershfield, Phys. Rev. B 43, 11586 (1991).
${ }^{10} \mathrm{~F}$. Flores and E. V. Anda (unpublished).

${ }^{11}$ M. Büttiker, Phys. Rev. B 40, 3049 (1989).

${ }^{12}$ P. L. Pernas, A. Martin-Rodero, and F. Flores, Phys. Rev. B 41, 8553 (1990).

${ }^{13}$ J. R. Kirtley, S. Washburn, and M. J. Brady, IBM J. Res. Develop. 32, 414 (1988); C. S. Chu and R. S. Sorbello, Phys. Rev. B 42, 4928 (1990).

${ }^{14}$ R. Landauer, Z. Phys. B 68, 217 (1987), and references therein.

${ }^{15}$ This has not been taken as a proper multiprobe measurement, since the inclusion of the ideal wire is assumed not to disturb the quantum-mechanical properties of the system. Nevertheless, some aspects of invasive measurements can be analyzed using such an external probe (Ref. 17).

${ }^{16}$ M. Büttiker, IBM J. Res. Develop. 32, 317 (1988).

${ }^{17}$ E. Tekman, PhD. thesis, Bilkent University, 1990.

${ }^{18}$ M. McLennan, Y. Lee, and S. Datta, Phys. Rev. B 43, 13846 (1991).

${ }^{19}$ E. Tekman and S. Ciraci, Bull. Am. Phys. Soc. 36, 411 (1991). 